\title{
ARC MAPPING: AN EFFECTIVE TOOL TO DETERMINE FIRE AREA OF ORIGIN IN FIRE INVESTIGATION
}

\author{
Muthuraman $\mathbf{N}^{1}$, Palani $\mathbf{P}^{2}$, Rajakarunakaran $\mathrm{S}^{\mathbf{3}}$ \\ ${ }^{1} P G$ Student, Mechanical Engineering, Kalasalingam University, Tamilnadu, India \\ ${ }^{2}$ Electrical Inspector, Tamilnadu Electrical Inspectorate, Tamilnadu, India \\ ${ }^{3}$ Senior Professor, Mechanical Engineering, Kalasalingam University, Tamilnadu, India
}

\begin{abstract}
The fire accidents often happen in the residential and commercial structures. The investigation of fire in the accident site is a complex process and there is a difficulty in identifying the cause of fire. The commonly encountered problem in fire investigation is the false statements of witness information, hidden evidences and insufficient data provided by the occupier of the premises. The investigation of fire accident done by the corresponding authority should follow a procedural method. The effective investigation results in accurate determination of cause of fire and other factors that led to the spread of fire. There are several investigation tools and techniques used for fire investigation such as Fire Pattern analysis, Heat and Flame Vector analysis, Depth of Char and Calcination Survey, Arc Mapping and Fire Dynamics. This paper discusses about the tool arc mapping which is used to identify the fire area of origin where fire involves electrical systems and this paper focuses on the possibility of arcs in the circuits which is energized and drawing power, energized and not drawing power and un-energized circuits. How the identification of arcs in the electrical line diagram of accident sites helps to determine the fire area of origin is also discussed. The differentiation between melting of wire and arcing between wires is illustrated as per NFPA 921. The possibility of number of arcs in the three phase system is more than single phase system. The different types of arc formations in the three phase electrical supply system are explained in this paper.
\end{abstract}

Keywords: Fire Investigation, Arc Mapping, Electrical line diagram, Arcing and Fire Area of Origin.

\section{INTRODUCTION}

The investigation of fire accident is done to identify the source of the ignition, cause of the fire and the prevention of fire accidents with similar cause in the future. The various tools used to determine origin and cause of fire as per NFPA 921, Guide for Fire and Explosion Investigation are Witness information, Fire Patterns (Depth of Char and Calcination Survey), Arc Mapping and Fire Dynamics [1]. Whenever the fire involves electrical circuit wiring, power supply chords and electrical appliances there is a chance of arcing if the circuit is live. The marking of arcs in the electrical wiring diagram of the site provides a pattern which is used to identify the fire area of origin which is discussed in this paper. This technique is based on the anticipated behavior of energized circuits faulting when the conductor's insulation covering is exposed to a spreading fire [1]. The arc mapping is used to find only the area of fire origin in the site and not the cause of the fire. Once the area of fire origin is identified, then the surroundings are analyzed for the source of ignition and the conclusion is arrived. As per Michel early in the fire and soon after the flames reach the various circuits, one by one short circuit accompanied by electric arcs will takes place. Such arcs damage the wires and this damage can be used by the investigator to reconstruct the course of the fire [3].

Whenever electrical systems are involved in the fire, generally it is said that electricity is the cause of the fire which is not the realistic situation. There is always a difference between electrical circuit initiated the fire and electrical circuits which is affected by fire. Not all burnt wires are cause of fire unless until it is proven strongly. Till now there is no proven theoretical basis or law which could say electricity is the cause. Research is going on this issue. As per Vytensis Babrauskas [5] discussed about the various testing methods used to differentiate between cause beads and victim beads and also the author listed the possible errors and disadvantages in testing methods of burnt wire in identifying the cause. The electricity as a cause are said by thorough analysis of the situation and considering all factors of ignition source. Even there is a chance, that the occupier of the accident premises site may remove some of main cause factors thereby the blame is on electricity as the cause. Arc mapping is a tool to identify fire area of origin and analysis should be made with the collected data and accident sites and cause can be determined. The un-energized wire when attacked by fire it only melts whereas energized wire forms an arc. Once the energized wires are attacked by fire the corresponding circuit breaker is tripped or the fuse is broken. As per Daniel the location of arcs are marked and origin area is identified based on the principle, the arc that is found furthest from the power source is the one which occurred first[6].

\section{RELATED WORK}

D Churchward et al [2] reveals about the benefits of arcing. The author said arc mapping can be used as a tool for fire investigation. The author discussed about the procedure for 
arc survey and the ways to differentiate between melting and arcing evidence and also the documentation to be done during arc survey. The author discussed about the possible errors that can be made during arc survey and said mainly about the cause determination which includes consideration of fuel, ignition source and ignition sequence. The contribution of arcing in fire and arcing as the fire cause factor and its possible conditions are also discussed by the author.

Nicholas [4] made a research on arc mapping. The author made a research to test the reliability of using the electrical wiring in a building to establish the origin of a fire. The test consists of furnished experimental compartment fires using repetitive room configurations and the result specimens are analyzed with various types of microscopy. The analysis of the data indicated that there is a high probability that arcing damage observed on electrical conductors occurred near to the fire's area of origin. The series of experimental fires validated the reliability of using the arc fault mapping methodology during the investigation of fire scenes. The author also mathematically validated the reliability of the arc mapping methodology and confirmed that the arcing events occurred irrespective to the fuel loads and fuel configuration within the compartment. The experimental tests where the arcing damage was used to suggest an area of origin of the fire was successful in all tests and illustrates the value of this methodology in scene investigation particularly when post flashover has occurred.

Michel Delplace et al [3] discussed that electrical short circuits help the investigator determine where the fire started and said that copper wires will survive with little damage in most fires. If this wiring was live during the fire, it will show damage by electric arcs. The author discussed about the characteristics of copper and the types of wire damage such as arc damages in wires, Alloying by chemical damage, heat damage and chemical damage. The author also explained the field procedure to do the arc survey in the accident site and the information to be collected prior to investigation. Once the arc survey is completed the point of origin should be traced by analyzing the arc sites and surrounding materials. The explanation for the concept is given with few examples and the interpretation of the collected information. Finally the author discussed about the possibility of electricity as the cause of the fire and how short circuits play a role in the fire initiation.

Daniel L [9] validated the arc survey in determination of fire origin. The author discussed about the definition of arc survey and the procedure for conducting an arc survey and the documentation of arc survey and the evidence gathering of arc survey. The author also discussed about the test burns conducted in compartment fire conditions. The author reveals that the spatial relationship of arc sites located in the structures helps the investigator to determine that where fire damaged electrical distribution system before the electricity was shut off.

\section{POSSIBILITY OF ARCS IN ELECTRICAL CIRCUIT}

An arc is a momentary flash of electric discharge that occurs between two conducting medium across a gap through a medium such as air, dust or charred insulation. The electrical wiring of residential or commercial circuits encounters arcing under various conditions when attacked by fire.

\subsection{Electrical Circuit Distribution}

The common electrical line diagram of a residential circuit would be as shown in figure 1 .

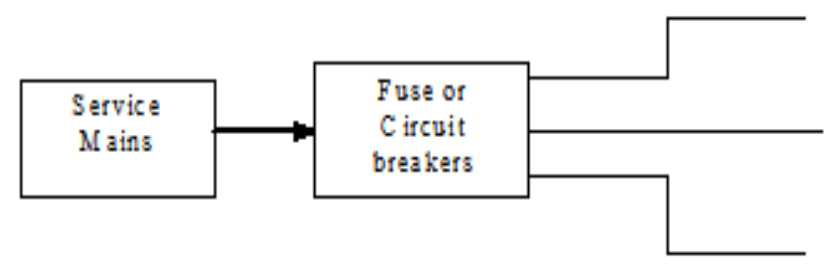

Fig -1: Electrical line diagram of a common residential circuit

Normally the electrical systems are connected such as starting from the utility transformer it is connected to the service mains of the premises and to the appliances via fuse or circuit breakers. The service is segregated in to different branch circuits and each branch circuit is connected to different sets of appliances. The concept of arc is that when an energised conductor is exposed to fire the insulation cover of the copper conductor breaks down and it becomes a semiconductor thereby electrons flow between the two conductors or one conductor and metallic surface or one conductor and ground which is found as arc. Once arcs are formed the circuit breaker is tripped or fuse is broken.

\subsection{Arcing}

The electrical service line diagram is as shown in figure 2 .

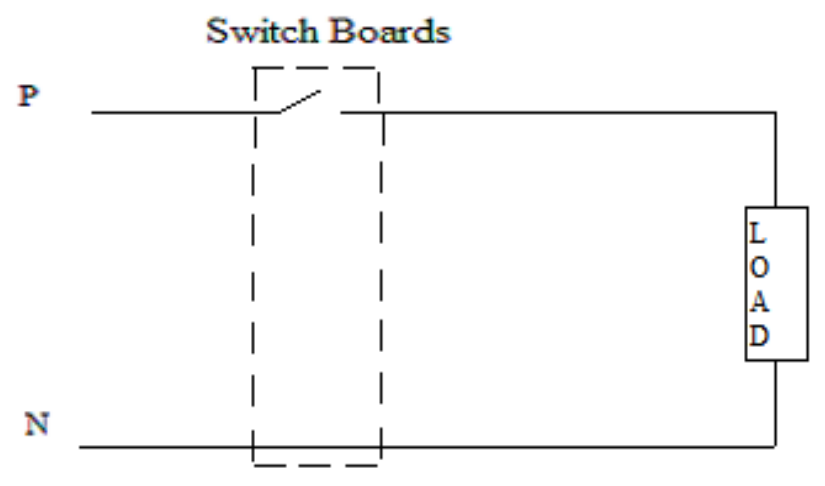

Fig -2: Electrical service line diagram

To understand the current flow path there is a distance between phase and neutral but in practical both are close and they are put in a conduit as shown in figure 3. 


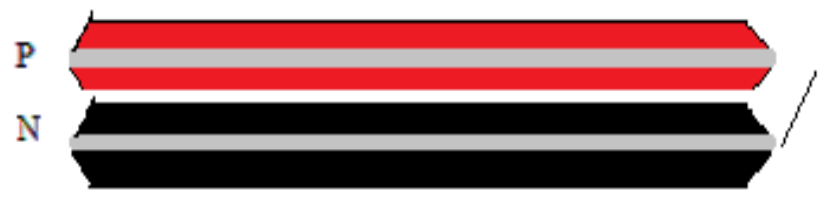

Fig -3: Wires lay in conduit pipes

If the wire is energized and when fire attacks, the insulation of two wires burns and conducts where a short circuit occurs and it is formed as an arc. The different response when fire attacks the wire is,

- Circuit is Live and Drawing Power: When the circuit is live and switch is on the arc may be found between service entrance and the switch board and also between switch boards to the load as the circuit is live all through the wiring connection

- Circuit is Live and not Drawing Power: This is the case where the circuit is energized till switch board but it is kept in open position. So when fire attacks the wiring position near the load there will be no effect of arc as there is no flow of voltage. But if the fire attacks the portion between service entrance and the switch board short circuit occurs and arc is formed

- Circuit is Alive: When the circuit is alive that is the main is shut-off or fuse broken there is no chance of arcing between service entrance, switch board and load. Only there is a chance in service from distribution transformer to service mains which is out of scope here

\subsection{Three Phase System}

The three phase systems in residential structures or commercial buildings also form an arc when attacked by fire. The three phase circuits are classified in to single phases for certain group of loads, in these cases the arcing is similar to that of discussed previously. If the load requires three phase supply then the wiring used for this load is 4 wire systems. Depending upon the load the three phase four wire system is used. The illustration of the formation of arc in three phase system is shown in figure 4.

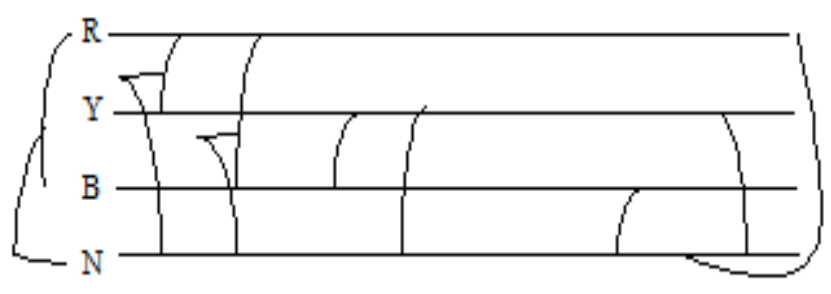

E

Fig -4: Three phase systems short circuit paths

There are several possibilities of short circuit in three phase system such as RY, YB, RB, RYB, RN, YN, BN, RYBN, RYN, YBN and RBN. There are seven other possibility with respect to earth similar to that of neutral. Thus in case of three phase system, if the fire attacks such wiring system multiple arcs will be found which needs to be depicted correctly.

\section{ARC MAPPING}

The arc mapping involves identifying all arcs in the accident site and plotting it in the electrical line diagram of the site plan and analysing the spatial relationship between them.

\subsection{Arc Survey}

An electrical arc survey is the identification and documentation of electrical arcs found in circuit wiring, power supply cords, or loads. The location of these arcs and their spatial relationship between them provides the fire area of origin. Before starting to find the arcs in the site there are certain details to be collected. The site plan of the accident premises is drawn and the full electrical lay out diagram is drawn which includes number of branch circuits, size of each conductor, connection to all loads and switch boards. The details about the fuse or circuit breaker condition are also collected. The arcs are identified by examining the conductors from the service entrance to the load for every branch circuit. The differentiation between melting and arcing should be known. Arcing makes damage localised between two conductors, a conductor and ground or between conductors and metallic surface whereas melting is widespread where NFPA 921 [1] provides several visual examples, a sample of melting is shown in figure 5 and arcing between conductors is shown in figure 6 .

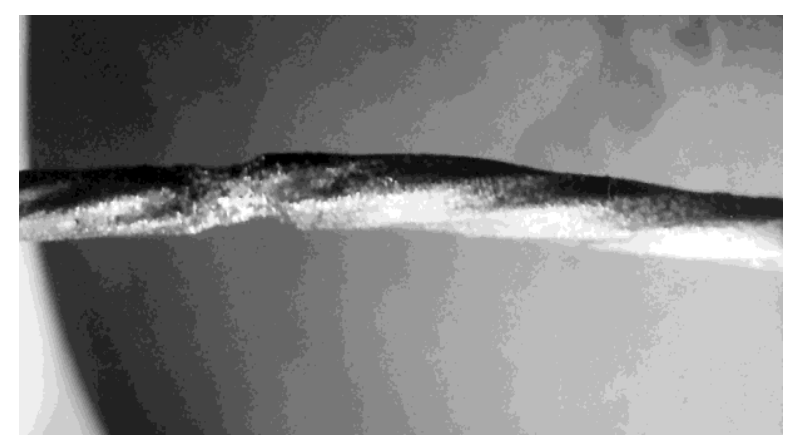

Fig -5: Stranded copper conductor in which melting by fire caused the strands to be fused together [1]

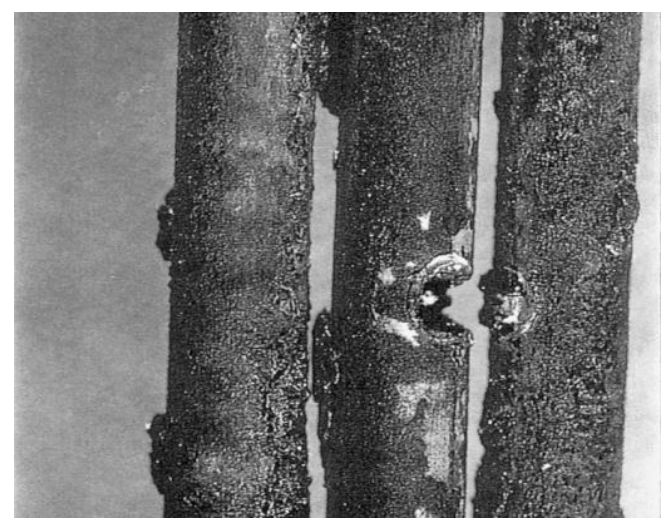

Fig -6: Spot arc damage to conductor caused by arcing through charred insulator [1] 
The identification of arcs can also be done by taking samples from the accident site and test the samples in laboratories as arcs or melt. The tests include Metallurgical analysis of copper and surface chromatography and several other tests are also available.

\subsection{Fire Area of Origin}

If there are multiple arcings found on a single branch or in all branch, it is principle that the arc which is found furthest from the power source is the one which occurred earlier in the fire which is illustrated in the figure 7.

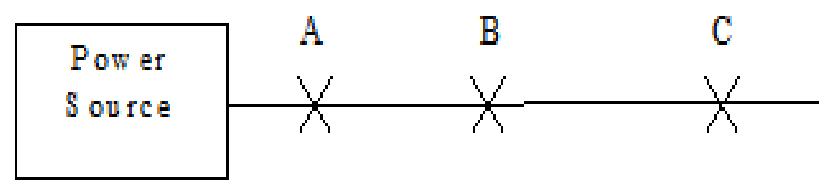

Fig -7: Earlier arcing illustration

In the above figure the arc $\mathrm{C}$ is the one which occurred earlier the reason because in case if the arc A or B occurred first then the current flow is interrupted and there is no further flow of voltage beyond that point. For example consider the following electrical line diagram as shown in figure 8 . There are four branch circuits from the service mains. The furthest arc is $\mathrm{F}$ in the fourth branch and the next furthest is the $\mathrm{C}$ in the third branch where these two arcs are nearby and this is the area where the fire started first. Then the source of ignition near the area should be considered for analysis.

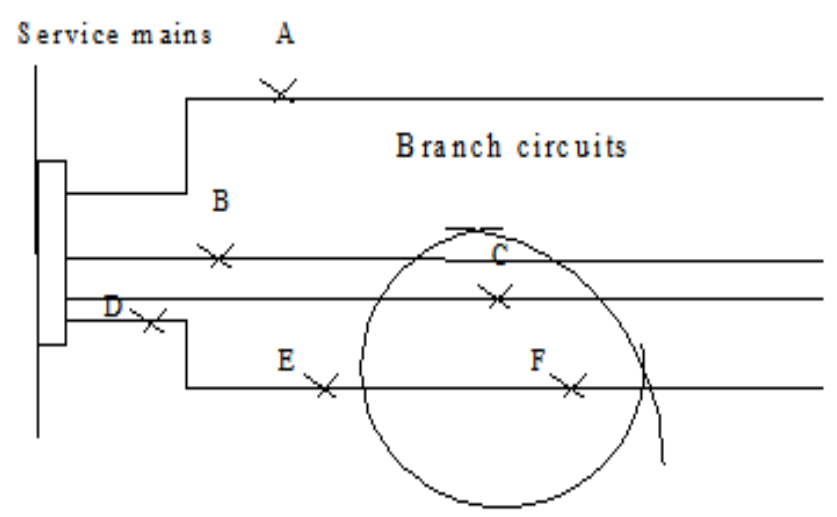

Fig -8: Layout of site with arc mapping

The path of the arcs shows the fire propagation from the area of origin to the final burning area. The electricity is shut off once the arcing tripped the circuit breaker or fuse or the fire fighters may shut it off for fire fighting. There is a chance that the arcing may be overcome by severe heat damage of heavy fire or the fire suppression medium used could also have destroyed the signs of arcs on the site.

\section{CONCLUSIONS AND FUTURE WORK}

In this paper, we presented the use of a tool arc mapping in determining the fire area of origin in fire investigations and the possibility of arc formations in the residential circuits at various power conditions. The differentiation between melting and arcing is discussed and the various types of arc formations in the three phase system is also discussed. The future work includes the application of arc mapping in a real time fire accident involving electrical circuits and to check for the results with other normal types of fire investigation methods.

\section{ACKNOWLEDGEMENTS}

I thank the Electrical Inspector Palani $\mathrm{P}$ working in Tamilnadu Electrical Inspectorate for providing me a direction towards fire investigations and need for investigation methods in electrical fires.

\section{REFERENCES}

[1]. National Fire Protection Association. NFPA 921 "Guide for Fire and Explosion Investigations", 2004 edition

[2]. Daniel L. Churchward, CFEI and Ryan M. Cox, CFI, CFEI, CFPS Kodiak Enterprises, Inc. USA, "THE 'BENEFITS' OF ARCING'

[3]. Michel Delplace and Eddie Vos, Consulting Engineers, "Electrical short circuits help the investigator determine where the fire started

[4]. Nicholas Carey and Niamh Nic Daeid (2010), Strathprints Institutional Repository, "Arc Mapping Research".

[5]. Babrauskas, V., Fires due to Electric Arcing: Can 'Cause' Beads Be Distinguished from 'Victim' Beads by Physical or Chemical Testing? pp. 189-201 in Fire and Materials 2003, Interscience Communications Ltd., London. [6]. Daniel L. Churchward, Ryan M. Cox \& David Reiter, PE, "Arc Fault Survey as a means to determine Fire Origin in Residential Structures"

[7]. Donald J. Hoffmann, Ph.D., P.E., C.F.I., Safety Engineering Laboratories, Inc, "Beyond Tea Leaves-Using Arc Mapping to Pinpoint Origin and Cause of a Fire"

[8]. Dr. Bernard Beland, Department of Electrical Engineering, University of Sherbrooke, "Considerations on Arcing as Fire Cause"

[9]. Daniel L. Churchward, Ryan M. Cox \& David Reiter, PE, "Arc Survey as a means to Determine Fire Origin in Residential Structures"

\section{BIOGRAPHIES}

Muthuraman N currently studying M.Tech Industrial Safety Engineering at Kalasalingam University and completed B.E degree in Electrical and Electronics Engineering at Sethu Institute of Technology in 2010. The research areas of interest include Investigation of Electrical Fires and identification of electricity is the cause or victim.

P.Palani currently working as a Electrical Inspector in Tamilnadu Electrical Inspectorate. Conducted Various Electrical Inspection and Fire Investigations involving electrical systems

Dr. S. Rajakarunakaran has obtained his Bachelors Degree in Mechanical Engineering from Madurai Kamaraj University, Madurai and Masters in Industrial Safety 
Engineering from the Regional Engineering College (presently known as National Institute of Technology), Thiruchirapalli. He received his $\mathrm{PhD}$ from the College of Engineering, Guindy (CEG), Anna University, Chennai in the field of Safety Engineering and Management. His expertise lies in the area of Safety Engineering, Condition Monitoring, Fault Diagnosis and Soft Computing Applications. 